\title{
MOD/R: A Knowledge Assisted Approach towards Top-Down only CMOS VLSI Design
}

\author{
L. Spaanenburg, M. Beunder, F.A. Beune, S.H. Gerez, B. Holstein \\ R.C.C. Luchtmeyer, J. Smit, A. van der Werf, H. Willems \\ Twente University of Technology, Department of Electronic Engineering \\ P.O. Box 217, 7500 AE Enschede, The Netherlands
}

\begin{abstract}
MOD / $R$ models all views on the design space in relations. This is achieved by eliminating the package constraints, as are apparent in PCB oriented hardware description languages. Assisted by knowledge engineering it allows for a top-down, mostly hicrarchical decomposition, virtually eliminating the need for bottom-up assembly.
\end{abstract}

\section{Introduction}

IC design is still based on a hierarchy of fabrication technologies and therefore on a hierarchy of packages as there are integrated circuits (ICs), thermal conduction modules (TDMs), printed circuit boards (PCBs), racks, cabinets, etc. Consequently, a range of $\mathrm{CAD}$ programs and data bases exists to accommodate these differing realizations.

This situation might have been acceptable in the early days of chip design, when the chip was nothing more than an odd collection of components put together by a physics-oriented specialist. Nowadays, however, it should be rejected for two reasons. First of all, the package limitation has become obsolete now that an entire system can be realized on a single chip or even a single wafer. Moreover, this limitation considerably restricts the possibility to use silicon efficiently. Secondly, the requirement that he or she should learn to deal with a wide range of design tools, slows down the education of the designer in an era when so many of them are needed

A fresh start is necessary, keeping in mind the intricacies of VLSI and aiming for a single design language and a single design environment. Such an approach, however, entails a new set of CAD tools tailored to these needs: MOD/R aims to provide these.

MOD/R is based on an enforced separated hierarchy, that eliminates process dependency from the initial specification and allows for a formal proof. By means of quality factors this specifica. tion is rearranged in order to get the best layout in a top-down fashion. At the back-end, knowledgebased module generators are used that are able to produce full custom designs. This virtually eliminates the need for cell libraries and totally climinates the need for various cell library versions, which provides an alternative look on the phenomenon of second-sourcing.

\section{Hierarchy}

The obvious way to solve a complex problem is to divide it into parts. Such a "divide-and-conquer" approach can only solve the problem, however, when the complexity of the sum of the parts is less than the complexity of the original problem. This is not always the case. For instance the IEEE$188 / 1978$ measurement interface standard was specified as a set of 10 separate functions [2]; their interrelations were so complex, however, that most of the initial realizations had at best minor bugs. This circuit was relatively small; for larger circuits the problems grow exponentially.

For this reason the notion of hierarchy and therefore abstraction has been introduced. The circuit is gradually divided into parts, where each division introduces a new but lower level of abstraction. Such a specification can be viewed as a tree, the root of which represents the most abstract specification and the leaves of which represent the least abstract one. Nothing is stipulated on the precise content of the intermediate specifications. Rather, it is assumed that the tree reflects the layout in a one-to-one correspondence.

In a separated hierarchy all computation as derived from the specification is confined to the leaf cells [3]. All intermediate cells reflect only an order of composition (assuming the existence of "flexible" or "gummy" cells). This separates bchavior from structure, thereby eliminating process dependency. The restrictions on the compound cells have to be relieved somewhat to allow for relabeling. The leaf cells are distinct, hence the need for logic collapsing arises: if several leaf cells each encapsulatc one and the same logic function, they are close to each other and they are used in different time intervals, they can be allowed to share the same hardware provided that their 1/O-signals are relabeled. The hardware overhead necessary for this relabeling (e.g. multiplexers and demultiplexers) is not considered to be part of the "computation" and is therefore permitted to be part of 
composite cells. Examples of different ways to specify a hierarchy are illustrated in Figure 1 (the sytem described is a controller for a read function).
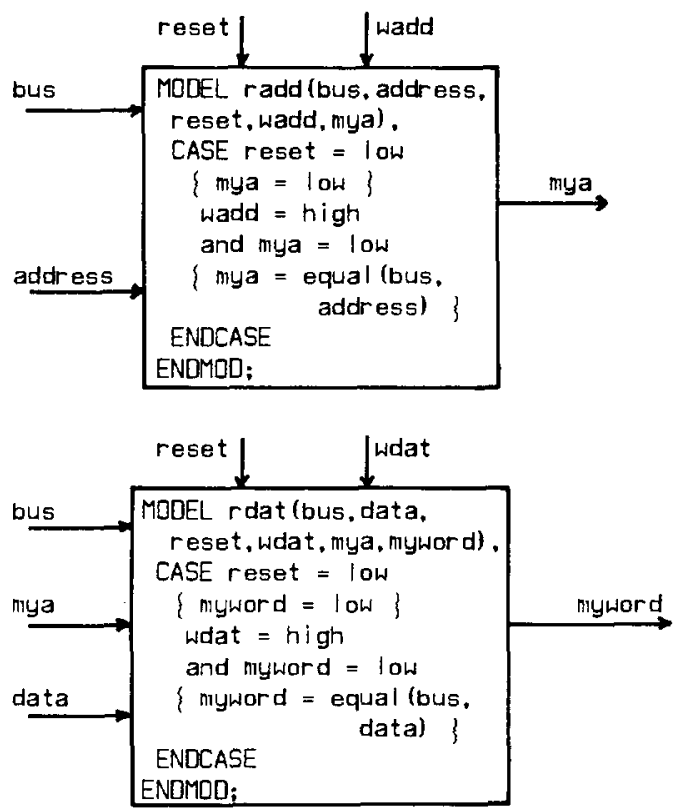

Figure $1 \mathrm{a}-$ Divide and conquer in specification.

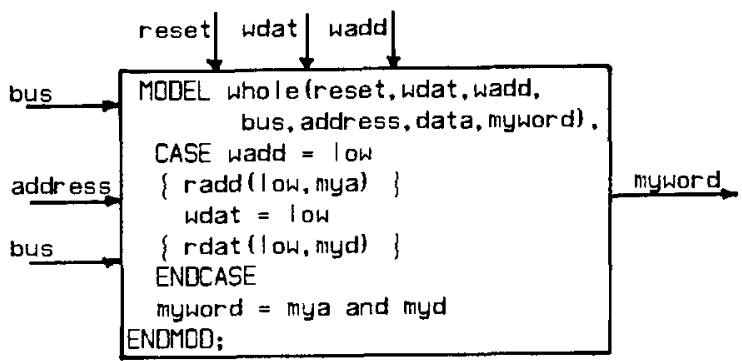

Figure 1b - Hierarchy in specification;

the last statement specifies a computation.

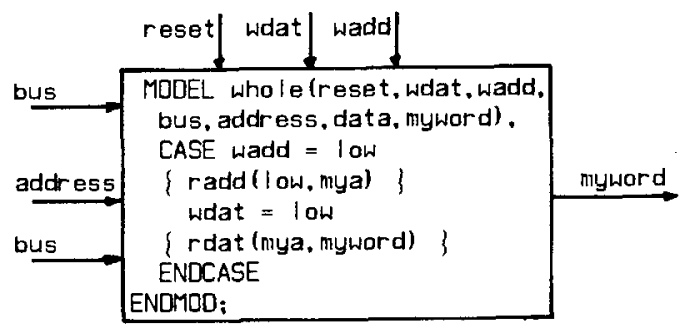

Figure 1c - Separated hierarchy in specification

MOD $/ R$ requires that a separated hierarchy is used for the complete specification of a chip. In fact, when a specification is validated to obey the requirements of a separated hierarchy, it can be proven to be correct rather than being simulated (which after all cannot guarantee correctness). For instance, the specification can be made in terms of state diagrams [1].
The design is entered in an SADT-like (Structured Analysis and Design Technique) graphical notation [4]. Every design part is represented as a rectangle with on the left hand side the incoming data signals, on the right hand side the outgoing data signals, on the upper side the control signals and on the lower side the environmental constraints. Each design part is associated with a document which describes its internal and external behavior, as well as its internal and external structure. As such, the graphical representation acts as the gateway to the design files. During the step-wise refinement the system is partitioned and more documents are generated. This ensures that design is always documen:od, which eases e.g. debugging. Figure 2 illustrates this notation.

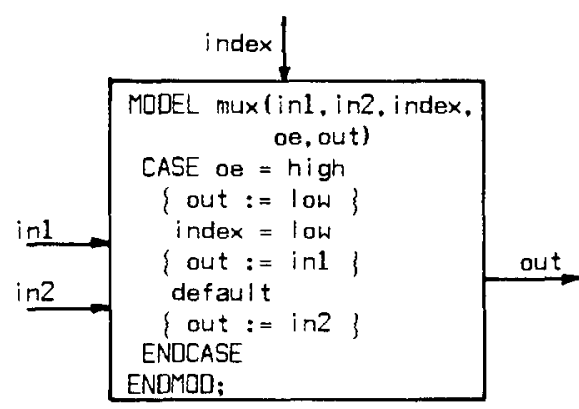

Figure 2 - Specification of a strobed 2 -input multiplexer.

Essentially there are three approaches to partitioning and hence three sets of labels that can be attached to design parts. The first distinction is between functional and distributive partitions. In a functional partitioning the cuts in the design are made perpendicular to the data flow; distributive partitioning, however, slices the design parallel to the data flow. The second distinction is between local and global partitioning. In a local partitioning all parts are kept within the data flow; in a global partition partitioning a second data flow is introduced. The third distinction is between logic and logistic: logic is an essential part of the data flow, whereas logistic concern power lines, clock signals etc. These three approaches in partitioning have a clear impact on the floor plan. MOD $/ \mathrm{R}$ operates on the different labels to format an initial specification such that it optimally fits in a proposed floor plan.

The partitioning of a data path is a straightforward action that is often encountered in practice. Recently it has been shown that a control path can be partitioned too. Figure 3 gives a schematic illustration of the different ways of partitioning.

\section{Quality factors}

Recently members of our group have concentrated on the use of qual ity factors as a means for the evaluation of the different steps in the topdown design process [5]

In the partitioning hierarchy the size of a module is of minor importance. However, within the floor plan hierarchy, the module size is of crucial impor- 

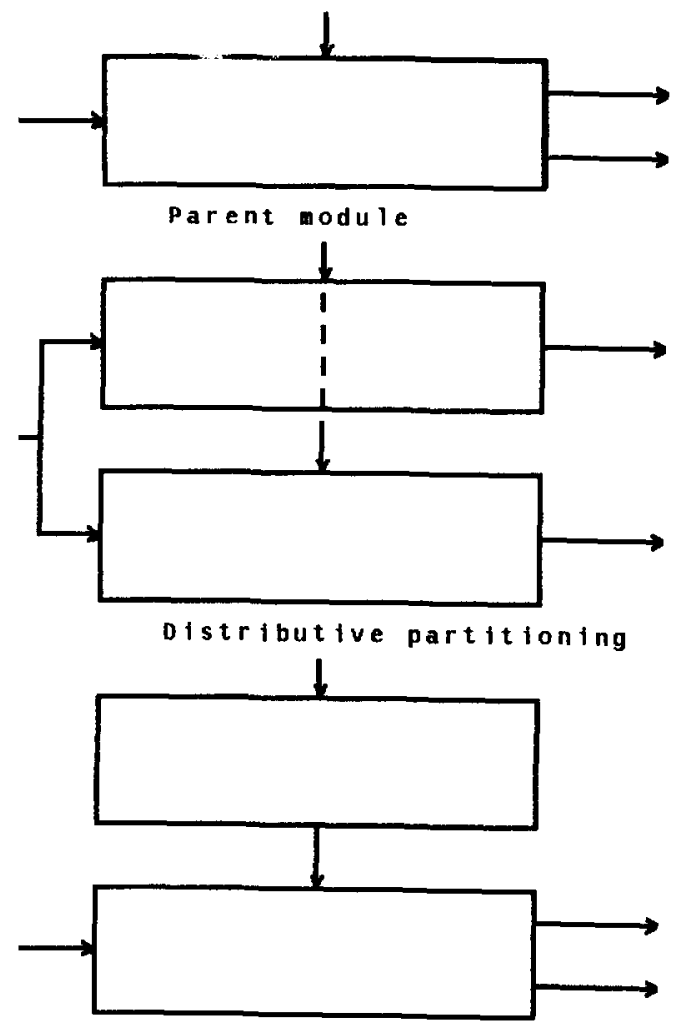

$\operatorname{logic/logistic}$ partitioning

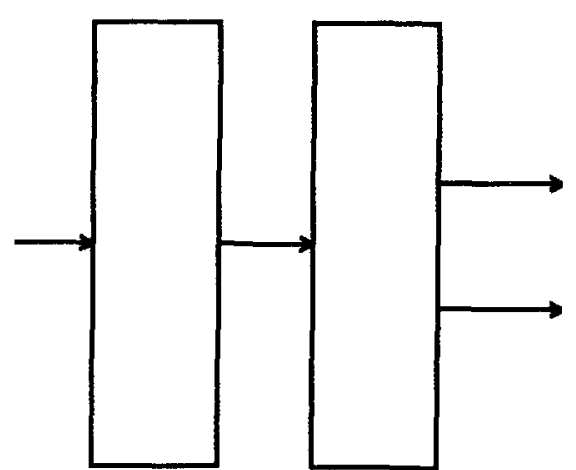

Functional partitioning

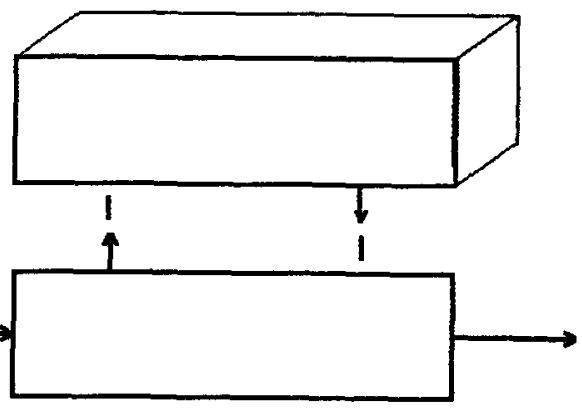

Global partitioning

Figure 3 - Various ways of partitioning tance. Because the absolute module placement basically depends on the average character of its terminals, the physical placenent factor (pI) is introduced. Each terminal connecting the module with an input, output or control signal of the main function to be realized (i.e. of the system io be integrated as a whole) contributes to the absolute placement of the module. The weight of the contribution depends on the kind of signal that is transported via a terminal (see Figure 4). For instance, clock signals in a synchronous circuit have a high weight because the delays introduced by long clock lines bring down the performance of the circuit as a whole.

\begin{tabular}{|l|l|}
\hline \multicolumn{2}{|l|}{ Placement factor per terminal } \\
\hline value & character \\
\hline 3 & Main function clock terminal \\
2 & Main function critical speed \\
& input output or contro! \\
& terminal \\
1 & Main function non-critical \\
& speed input output or \\
& control terminal \\
0 & Local diagram terminal \\
\hline
\end{tabular}

Figure 4 - Physical placement factors

The module connectivity factor is related to modules that have a main function input, output or control signal in common. It is defined as the sum of the separate signal weight factors (see Figure 5).

\begin{tabular}{|l|l|c|}
\hline \multicolumn{2}{|l|}{ Signal Weight Factors } \\
\hline Signal Class & Subclass & Weight Factor \\
\hline $\begin{array}{l}\text { Control } \\
\text { Signals }\end{array}$ & $\begin{array}{l}\text { Clock Signals } \\
\text { others } \\
\text { Signais }\end{array}$ & $\begin{array}{c}4 \\
\text { Critical } \\
\text { speed, sync. } \\
\text { signal values } \\
\text { Non-critical } \\
\text { speed, sync. } \\
\text { Critical } \\
\text { speed, sync. } \\
\text { Non-critical } \\
\text { speed, async. }\end{array}$ \\
\hline
\end{tabular}

Figure 5 - Signal weight factors

The quality of the resulting floor plan is evaluated by considering three factors. The contact defect factor (cdf) of a floor plan is the sum of values of all module interconnections which have to cross other module domains. If a connection between two modules has to cross another module, the value of the interconnection is taken into account. The total length of wires (tlw) interconnecting the different modules is also of importance. The area occupied by wiring will be proportional to this quality factor. The wire usage factor (wf) is the sum of the quotients of signal weight and the length of the interconnection via which the signal is transported. The best quality is found when cdf and tlw are minimal and wf is maximal. 


\section{A theoretical example}

The main objective of this example is the demonstration of the use of the proposed quality factors. The design process starts with the top-level specification of the function (see Figure 6).
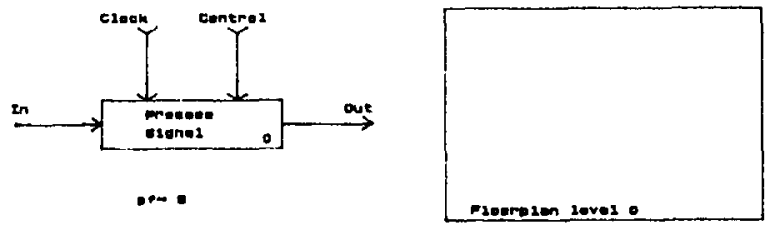

Figure 6 - Top level specification

The logic and logistics parts are isolated (Figure 7)

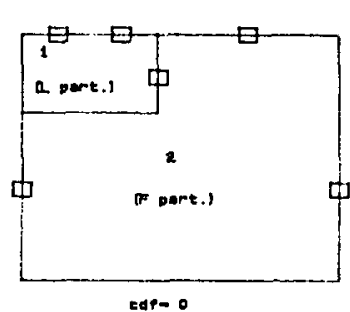

Floordian Lovel 1

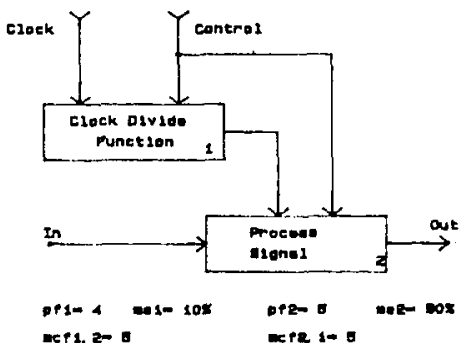

Figure 7 - Results of the first partitioning operation
The next step is the logic/logistic partitioning of module 1 and the functional partitioning of module 2 (Figures 8 and 9 )

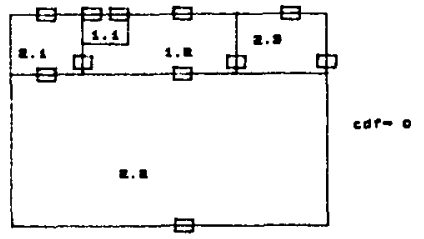

Figure 9 - Floorplan (second level)

Then only the modules 2.2 and 2.3 need a further partitioning. During the above steps, connections become visible which influences the structure of the floor plan. What makes good sense in the initial floor plan, may not be a good choice when more data are available. Quality factors may indicate that some decisions are questionable. This explains why modules change places in the hierarchical range of floor plans. Another point to be raised here is the clear visibility of the partitioning strategy in the relative location of the modules. For instance a logic and logistic part are always laid out in an orthogonal manner (Figure 10)
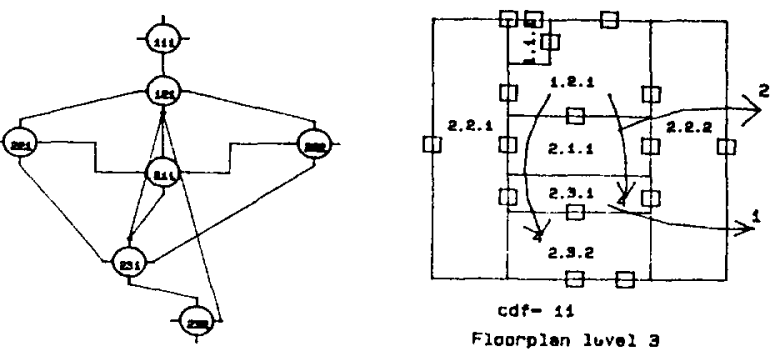

Figure 10 - Communication grpah and third level floor plan

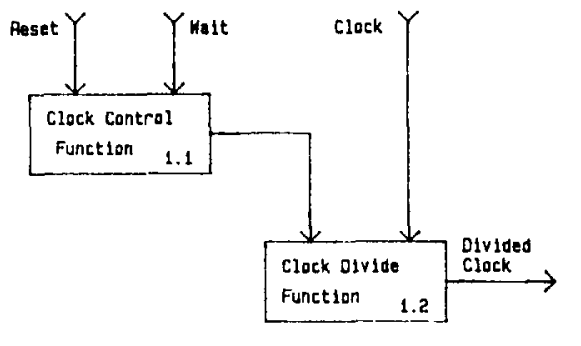

pf 1. 1- 2

as $1.1-20 x$

ect 1.1.1.2-

Df1.2- 3

$051.2-60 x$

mef1.2 1.1-

acf 1.2.2.1. 3- 4

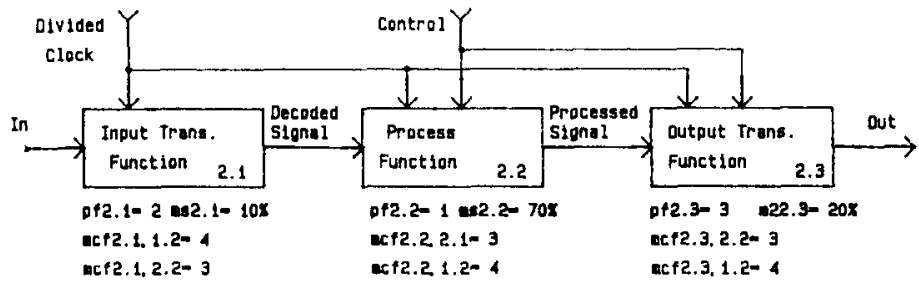

Figure 8-Result of the second partitioning operation 


\section{Module and cell generation}

MOD/R contains three module generators tailored for specific tasks: ODAC, RAC and miniBFN.

The first one, DDAC (One-Dimensional Array Compiler) is standard celi oriented. It allows standard cells to be connected in a row and to be wired externally. The result is then stored as a new standard cell. ODAC not only supports standard cells, but also gate arrays, cell arrays and Weinberger arrays configured according to the description given by a separate image compiler.

The alternative is RAC (Regular Array Compiler). From a library of basic cells it composes a twodimensional layout in a way similar to current day PLA compilers. However, only cells performing a certain computation are used; wiring cells are generated from the terminal description. This concept limits the number of cells to be dealt with considerably; in a PIA, for instance, $30 \%$ of the cells in use do not contribute to the functionality of the PLA. The input to RAC is a floor plan description in the form of a regular expression. This not only results in an casy interface to the MOD/R specification language, but also in the capability to generate any regular two-dimensional array

Most cells in a cell library look alike. The differences between some of them are sometimes marginal. This would not have been necessary, if cells would be generated from a generalized image. For instance in a cell library a lot of cells are latches with small variations. They could have been generated from a single general-purpose latch. A part of $M O D / R$, called miniBEN, is a set of rules written in PROLOG, that generates a mask layout from an example layout according to the redesign principle; the output is a cell layout that fits optimally inside its environment. miniBEN has more or less the same objectives as TOPOLOGIZER [6]

First of all miniBEN performs a rough geometric placement and a first assignment of wires to mask layers from the specification of the target cell area and terminal placement. In subsequent steps the cell is compacted by replacing devices, removing and inserting contact windows and changing wire layers and connections.

miniBEN has three sets of rules: process rules, conmion sense rules and expert rules. Process rules describe the basic set of elements to be transistors and contact windows and the way these are related to wires on the various layers. The common sense rules manipulate the masks in order to remove spurious contact windows, change wire layer levels, rotate design elements, etc. In other words, they act on the layout as any layman with common sense would do. The expert rules are the most comprehensive as they require the knowledge extracted from the experienced designer

\section{Discussion}

MOD/R strives for top-down only VLSI design. Some of the tools are still in an experimental stage; other parts, however, such as the one- and twodimensional cell generators and the quality factors are already usable. What is still needed is the extension of the expert rules of miniBEN and the linking of the software packages on a graphic interface. A trial run has been made on a chip that performs a function within the PRX/D digital switching system according to CCITT recommendation G741. The floor plan of the original chip, that was designed using conventional tools is shown in Figure 11; figure 12 show the new one. It measures $30 \mathrm{sq}$. mm. excluding peripherals. Our floor plan gives rise to about the same chip. However, it was designed in just a couple of weeks and has has a considerably lower contact defect ratio. One may therefore expect a faster chip. What is more important, it shows that MOD/R is capable of coping with complexity in a balanced way.

\section{Acknowledgements}

MOD $/ \mathrm{R}$ is a research project that relates to MoDL, a hardware description language that is being developed as part of the ICD work station [7] (the description in the Figures 1 and 2 is given in MoDL). ICD in turn is a project that runs under the EEC Microelectronics Regulation. MOD/R also draws on the T-DARR design environment of 1 st Silicon Works Almelo (The Netherlands) [8].

\section{References}

(1) Spaanenburg, L. et al. - "A Methodology for the Fast and Testable Implementation of State Diagram Specifications", IEEE Journal of Solid State Circuits, SC-20(2), pp 548-554, April 1985.

[2] 'IEEE Standard digital interface for programmable instruments", (IEEE Service Center, 445 Hoeslane, Piscataway, N.J., USA, 1978)

[3] Rowson, J., "Understanding Hierarchical Design", PhD thesis (California Institute of Technology, Pasadena, CA, USA, 1981)

[4] "An Introduction to SADT, Structured Analysis and Design Technique". SoftTech Publications, MA, USA, February 1976)

[5] Beunder, M.A., "A Partitioning Strategy for VLSI Design Based on the Use of Quality Factors". MSc. Thesis (Twente University of Technology, The Netherlands, May 1985).

[6] Kollaritch, P.W and Weste, N.H.E, "TOPOLOGIZER: An Expert System Translator of Transistor Connectivity to Symbolic Layout", IEEE Journal of Solid State Circuits, SC-20(3), pp 799-804, June 1985.

17] Smit, J. et al., "Definition of the Syntax and Semantics of the Modelling and Design Language MoDL", Internal Report, Twente University of Technology, March 1985.

[8] Gradenwitz, P.G.M. and Spaanenburg, L., "A Gate Array Optimized for Switch Level CMOS Logic with Guaranteed Timing and Backup Supply", Digest 5th ICSSI, London, November 1985. 


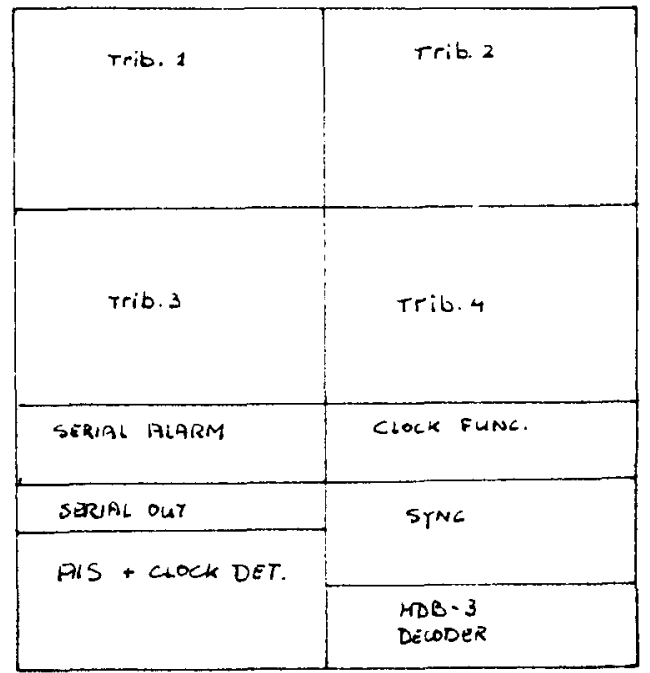

Figure 11 - The original floor plan of the OQ 2004

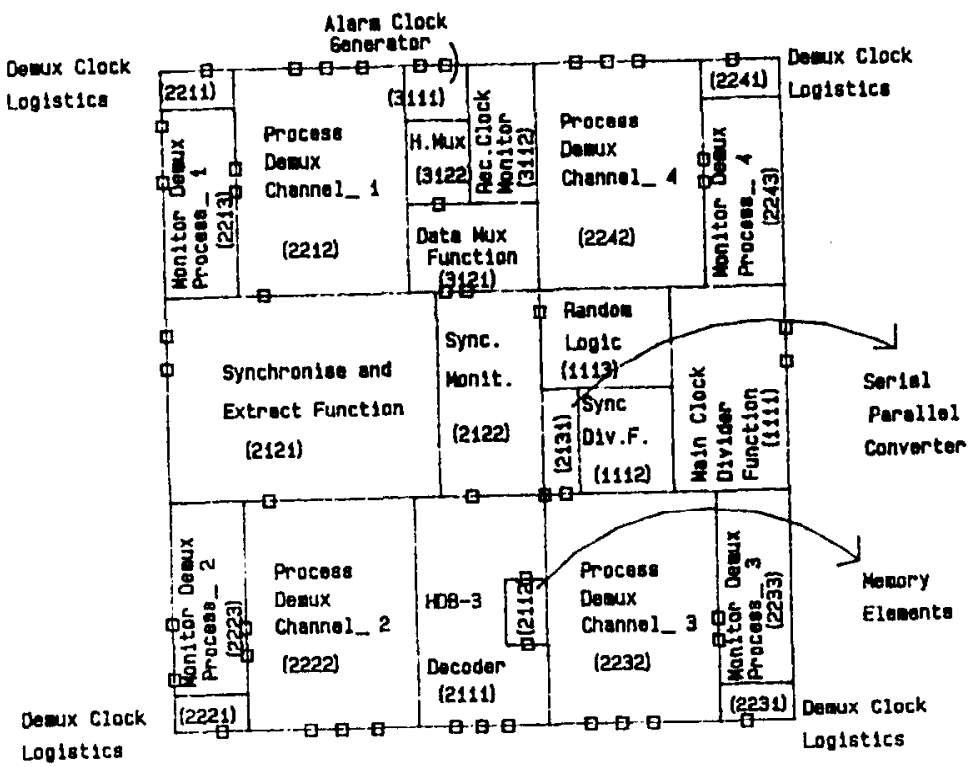

Figure 12 - Final (new) floor plan of the $O Q 2004$ 